\title{
Rotation-induced lithium depletion of solar-type stars in open stellar clusters
}

\author{
R. Tschäpe and G. Rüdiger \\ Astrophysikalisches Institut Potsdam, An der Sternwarte 16, 14482 Potsdam, Germany \\ Received 1 March 2000 / Accepted 26 March 2001

\begin{abstract}
We look for a correlation between Lithium abundance and rotation period in solar-type stars (G0... K0). The result is not unambiguous. Stars in young open clusters (IC 2391, IC 2602, IC 4665, $\alpha$ Per) do not show any correlation, in the Pleiades cluster a correlation is present: the faster the rotation the less the Lithium depletion. Moreover, there is statistical evidence, that at least in the Pleiades and in the $\alpha$ Persei cluster the abundance spread at fixed color in cool stars could be due to rotation. The use of the inverse Rossby number instead of the rotation rate does not improve on the Li-rotation correlation.
\end{abstract}

Key words. stars: abundances - stars: evolution - stars: rotation - open clusters and associations: individual: Pleiades, Hyades, $\alpha$ Per, IC 2391, IC 2602, IC 4665

\section{Introduction}

Lithium (Li) at the surface of solar-type stars is a tracer for mixing processes in the deep stellar interior and its photospheric abundance is used for testing stellar models (besides the measurements of solar neutrino flux and asteroseismology data). However, it must be presumed that the initial $\mathrm{Li}$ abundance is known and that furthermore $\mathrm{Li}$ is destroyed by nuclear burning only. This, of course, set aside speculations about substantial Li production in flares (Livshits 1997) as well as apparent surface Li depletion as an artefact of NLTE line formation (Carlsson et al. 1994; Stuik et al. 1997).

Some Li depletion takes place already in pre-main sequence (PMS) stars. It increases with decreasing mass and - at fixed mass - with increasing metallicity. After 50-80 Myr the G stars have just arrived on the ZAMS (Prosser 1992; D'Antona \& Mazzitelli 1994; Randich et al. 1998). But this PMS Li loss ( 0.3 dex, Pinsonneault 1997) is by far not enough to explain the Li depletion in late main-sequence stars.

For solar-type stars two facts are to be explained: (1) the ubiquitous deficiency of $\mathrm{Li}$ in the Sun, in $\alpha$ Cen as well as in late-type cluster stars at different ages and (2) the large dispersion in Li abundance for stars at fixed color (or $T_{\text {eff }}$ ) in some open clusters.

There are observational indications that rapidly rotating stars preserve lithium better than slow rotators of the same mass (Balachandran et al. 1988, 1996;

Send offprint requests to: R. Tschäpe,

e-mail: rtschaepe@aip.de

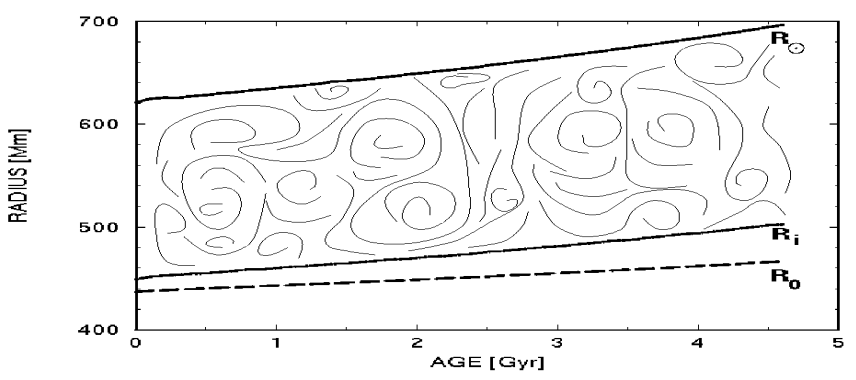

Fig. 1. The secular increase of the radial extent of the solar convection zone in the past. $R_{i}$ is the radius of the bottom of the convection zone while $R_{0}$ displays the lithium burning zone with a temperature of $2.6 \times 10^{6}$ K. After Stix \& Skaley (1990).

Soderblom et al. 1993; Jones et al. 1997; Randich et al. 1997; Stauffer et al. 1997; Krishnamurthi et al. 1998; García López et al. 1994). This result was first found by $v \sin i$ measurements and was therefore subject to statistical assumptions concerning the unknown inclination angle $i$. Meanwhile there are photometrically determined rotation periods for stars in several open clusters (e.g. Prosser et al. 1993a,b; Allain et al. 1996; Krishnamurthi et al. 1998; Barnes et al. 1998). Using the Prosser-Stauffer archive (Prosser \& Stauffer 1998) we address the question whether there are observational hints that Li depletion in solar-type stars is inhibited by rotation and what parameter, rotation period or Rossby number, best mirrors such a possible Li-rotation connection.

King et al. (2000) have also investigated a possible correlation of $\mathrm{Li}$ abundance with rotation in Pleiades stars with $4500<T_{\text {eff }}<5500 \mathrm{~K}$ using the technique of 
Table 1. Properties of open clusters. The last column gives the number of stars with photometric periods and Li abundances (source: Prosser \& Stauffer 1998).

\begin{tabular}{lllrcr}
\hline \hline Cluster & {$[\mathrm{Fe} / \mathrm{H}]$} & $E(B-V)$ & $d(\mathrm{pc})$ & age (Myr) & number \\
\hline IC 2602 & 0.0 & 0.04 & 150 & 30 & 15 \\
IC 2391 & 0.0 & 0.04 & 150 & 30 & 7 \\
IC 4665 & 0.0 & 0.18 & 350 & 30 & 8 \\
$\alpha$ Per & 0.05 & 0.1 & 165 & 50 & 22 \\
Pleiades & 0.034 & 0.04 & 127 & $80-100$ & 36 \\
Hyades & 0.127 & 0.0 & 42 & 600 & 23 \\
\hline \hline
\end{tabular}

detrending $\mathrm{Li}$ abundance determinations. They analyzed the deviations from a temperature-dependent mean $\mathrm{Li}$ abundance in a $\log N(\mathrm{Li})-T_{\text {eff }}$ diagram. They found some few Li-rich Pleiades stars with nevertheless slow rotation.

As in the King et al. (2000) paper we exclude known binaries. The reason is that tidally locked binaries in the Hyades have significantly higher Li abundances than single stars (Thorburn et al. 1993; Deliyannis et al. 1994). However, according to Ryan \& Deliyannis (1995) two Pleiades binaries do not have an abnormally high Li abundance as compared to single stars.

The theoretical background of the present study is the following. In solar-type stars Li burning becomes significant in view of the solar age at a temperature of about $2.6 \times 10^{6} \mathrm{~K}$ which is realized at a depth denoted with $R_{0}$ in Fig. 1. But the lower edge of the convection zone in the Sun is only about $1.2 \times 10^{6} \mathrm{~K}$. In order to explain the observed depletion, lithium must be transported from the convection zone (with its bottom $R_{i}$ ) to this hotter layer. There is a gap of about $20000 \ldots 40000 \mathrm{~km}$ between $R_{i}$ and $R_{0}$ (Fig. 1). The effect must be small, however, in order to allow for some lithium in the solar atmosphere even after 4.6 Gyr.

The question is how the chemicals are able to cross this gap. One possibility is overshooting convection (Ahrens et al. 1992; Blöcker et al. 1999; Schlattl \& Weiss 1999) another one is (highly) anisotropic turbulence stabilized by the radial temperature gradient (Zahn 1992; Vincent et al. 1996). In both cases the resulting anisotropies are opposing and so is the influence of the basic stellar rotation. Rüdiger \& Pipin (2001) have computed the rotational influence in the case of a turbulence field without any radial component. Only due to the Coriolis force the flow pattern obtains radial components in correlation with the horizontal intensities. The model calculations lead to the overall result that the $\mathrm{Li}$ transport through the layer below the convection zone (the "tachocline") is the less effective the faster the stellar rotation is ${ }^{1}$. The effect will be verified here by samples of stars of the same age and the same metallicity but with different rotation rates, i.e. G-type stars in young and intermediate-age clusters.

1 This is due to the rotational quenching of the eddy diffusivity, see Boubnov \& Golitsin (1995).
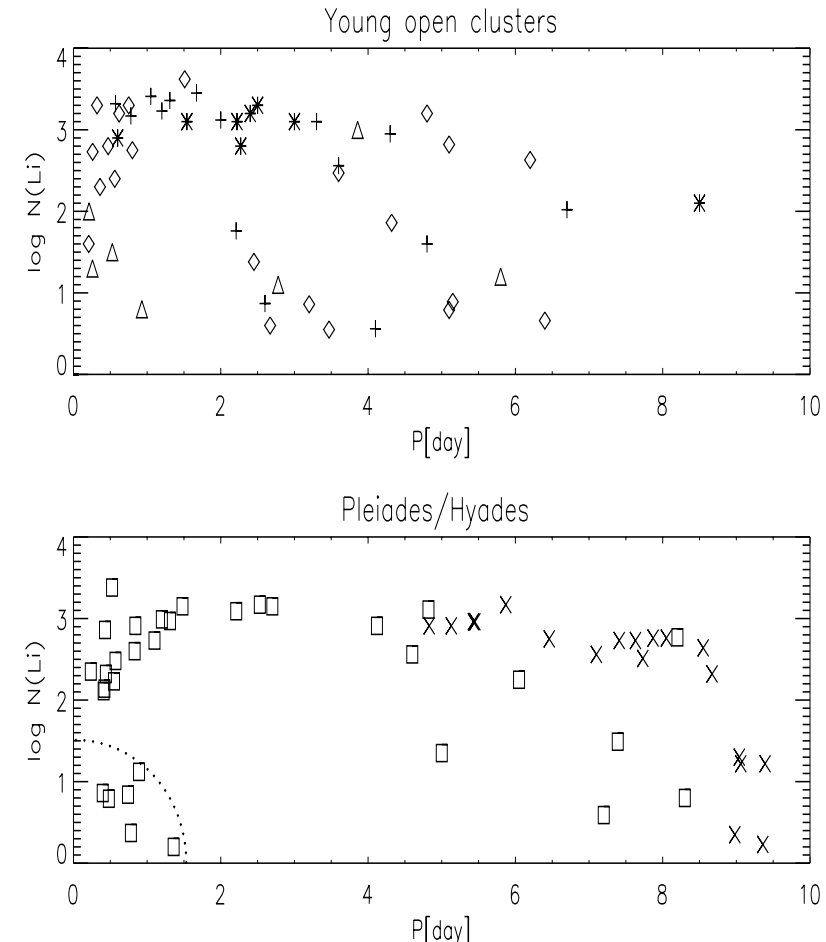

Fig. 2. Li abundance vs. rotation period, $P_{\text {rot }}$, for stars from the Prosser-Stauffer archive (Prosser \& Stauffer 1998). Top: "young" open clusters (age 30-50 Myr), bottom: "old" clusters (age 100-600 Myr). Symbols used are: (top) $\triangle$ (IC 2391), $+($ IC 2602), * (IC 4665), $\diamond(\alpha$ Persei); (bottom) $\square$ (Pleiades), $\times$ (Hyades). Encircled: fast rotating Li-poor stars.

\section{Observational basis}

Table 1 gives the numbers of stars with photometric periods and $\mathrm{Li}$ abundances available from the ProsserStauffer archive (Prosser \& Stauffer 1998). Figure 2 shows the $\mathrm{Li}$ abundance as a function of rotation period $P_{\text {rot }}$ (the symbols refer to different clusters; see caption).

No definite correlation can be determined from this data set at all. However, a group of fast rotating $\left(P_{\text {rot }}<\right.$ $1.5 \mathrm{~d}) \mathrm{Li}$-poor stars $(\log N(\mathrm{Li})<1)$ in the Pleiades is visible (Krishnamurthi et al. 1997). These stars have spectra between $\mathrm{K} 4$ and $\mathrm{K} 8$. We note the rotation period gap between 3 and 4 days.

Figure 3 demonstrates two well known facts, namely (i) the dependence of the photospheric $\mathrm{Li}$ content on color, i.e. on effective temperature and mass, respectively, and $(i i)$ the large spread in $\mathrm{Li}$ abundance, especially at $B-V \sim 1$, in the $\alpha$ Persei Cluster and in the Pleiades. The spread at a given $B-V$ indicates that mass, metallicity, and age do not uniquely determine a star's surface Li abundance. There must be an additional parameter (cf. Pinsonneault 1997). It is to be noted that the measurement error of an abundance estimate is $0.1 \ldots 0.15$ dex only.

In a second step we limit our discussion to solar-type stars, i.e. to stars within the spectral range G0...K0. Besides that, known binaries have been discarded.

There is only one solar-type star in the IC 2391 cluster. We have to discard this cluster. 
Table 2. Stars in open clusters with known $P_{\text {rot }}$ and Li abundances within the spectral range G0...K0. Spectral classes marked by $*$ are derived from reddening-corrected $B-V$ values according to Allen (1991). "<" indicates upper limit.

\begin{tabular}{|c|c|c|c|c|c|c|}
\hline$\overline{\bar{V}}$ & $B-V$ & $\begin{array}{c}P_{\text {rot }} \\
(\mathrm{d})\end{array}$ & 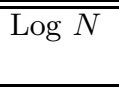 & $\begin{array}{c}v \sin i \\
\left(\mathrm{kms}^{-1}\right)\end{array}$ & 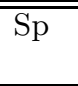 & " Name \\
\hline \multicolumn{7}{|c|}{ IC 2602} \\
\hline 11.86 & 0.82 & 1.31 & 3.36 & 34 & G8* & R59 \\
\hline 10.60 & 0.95 & 6.7 & 2.02 & 10 & G5 & B134 \\
\hline 10.52 & 0.65 & 0.57 & 3.31 & 93 & G0 & B102 \\
\hline 11.07 & 0.68 & 3.3 & 3.10 & 12 & $\mathrm{G} 4^{*}$ & $\mathrm{R} 66$ \\
\hline 10.92 & 0.69 & 4.3 & 2.95 & 11 & $\mathrm{G} 4^{*}$ & W85 \\
\hline 10.89 & 0.64 & 1.05 & 3.41 & 49 & G0 & B120 \\
\hline 10.70 & 0.62 & 1.67 & 3.45 & 30 & $\mathrm{G} 2 *$ & $\mathrm{R} 83$ \\
\hline 10.31 & 0.69 & 2.0 & 3.12 & 14 & G4 & B132 \\
\hline 11.73 & 0.87 & 1.20 & 3.23 & 12 & G9* & $\mathrm{R} 95 \mathrm{~A}$ \\
\hline \multicolumn{7}{|c|}{ IC 4665} \\
\hline 12.65 & 0.77 & 1.54 & 3.1 & 34 & G0 & $\mathrm{P} 27$ \\
\hline 13.68 & 0.92 & 3.0 & 3.1 & 17 & K0 & P71 \\
\hline 13.70 & 0.89 & 2.5 & 3.3 & 16 & G8 & P75 \\
\hline 14.26 & 1.01 & 8.5 & 2.1 & 10 & K0 & P94 \\
\hline 14.37 & 1.06 & 2.27 & 2.8 & 21 & K0 & P100 \\
\hline 13.08 & 0.86 & 2.22 & 3.1 & 25 & G5 & $\mathrm{P} 150$ \\
\hline 13.52 & 0.91 & 2.4 & 3.2 & 17 & K0 & $\mathrm{P} 155$ \\
\hline \multicolumn{7}{|c|}{$\alpha$ Persei } \\
\hline 11.69 & 0.79 & 0.60 & 3.05 & 87 & $\mathrm{G} 7 *$ & HE520 \\
\hline 11.43 & 0.73 & 3.6 & 3.44 & 15 & G6* & HE601 \\
\hline 11.66 & 0.82 & 0.8 & 2.75 & 61 & G8* & HE622 \\
\hline 10.59 & 0.57 & 0.75 & 3.30 & 71 & G0* & HE684 \\
\hline 12.08 & 0.87 & 4.8 & 2.89 & $<10$ & $\mathrm{~K} 0^{*}$ & AP 97 \\
\hline 12.06 & 0.81 & 0.32 & 3.30 & 160 & G8* & AP118 \\
\hline 12.38 & 0.89 & 0.26 & 2.73 & 170: & $\mathrm{K} 0^{*}$ & APX158 \\
\hline \multicolumn{7}{|c|}{ Pleiades } \\
\hline 10.93 & 0.72 & 8.2 & 2.77 & $<7$ & G4 & HII2341 \\
\hline 10.73 & 0.69 & 4.12 & 2.91 & $<7$ & G1 & HII152 \\
\hline 9.57 & 0.62 & 2.70 & 3.15 & 13 & G0 & HII739 \\
\hline 11.10 & 0.75 & 1.31 & 2.97 & 36 & G8 & HII1032 \\
\hline 9.70 & 0.55 & 1.2 & 2.99 & 50 & F9 & HII727 \\
\hline 10.13 & 0.62 & 1.1 & 2.73 & 45 & F9 & HII708 \\
\hline 11.57 & 0.84 & 0.84 & 2.91 & 19 & G8 & HII345 \\
\hline 12.18 & 1.00 & 0.524 & 3.38 & 68 & G8 & HII1136 \\
\hline 12.69 & 1.19 & 0.428 & 2.86 & 94 & K0 & HII625 \\
\hline 11.84 & 0.88 & 6.553 & 1.94 & $<7$ & G8* & HII34 \\
\hline 10.31 & 0.60 & 2.21 & 3.09 & 22 & G0* & HII 2786 \\
\hline 12.65 & 1.01 & 5.0 & 1.35 & 8 & G8* & HII2741 \\
\hline 10.79 & 0.70 & 4.2 & 2.69 & $<7$ & G1 & HII293 \\
\hline \multicolumn{7}{|c|}{ Hyades } \\
\hline 7.47 & 0.57 & 5.45 & 2.96 & & G0 & VB31 \\
\hline 7.85 & 0.61 & 7.41 & 2.73 & & G1 & VB73 \\
\hline 7.94 & 0.63 & 8.55 & 2.64 & & G1 & VB97 \\
\hline 8.12 & 0.66 & 8.67 & 2.32 & & G2 & VB64 \\
\hline 8.66 & 0.74 & 9.04 & 1.3 & & G8 & VB92 \\
\hline 8.63 & 0.74 & 9.39 & 1.22 & & G9 & VB26 \\
\hline 9.15 & 0.82 & 8.98 & 0.35 & & K0 & VB21 \\
\hline 8.96 & 0.83 & 11.38 & $<0.3$ & & K0 & VB79 \\
\hline
\end{tabular}

Table 2 contains data of these solar-type stars according to their membership to the young open clusters IC 2602, IC 4665, $\alpha$ Per and to the older ones, i.e. Pleiades and Hyades. The photometric values are not corrected for
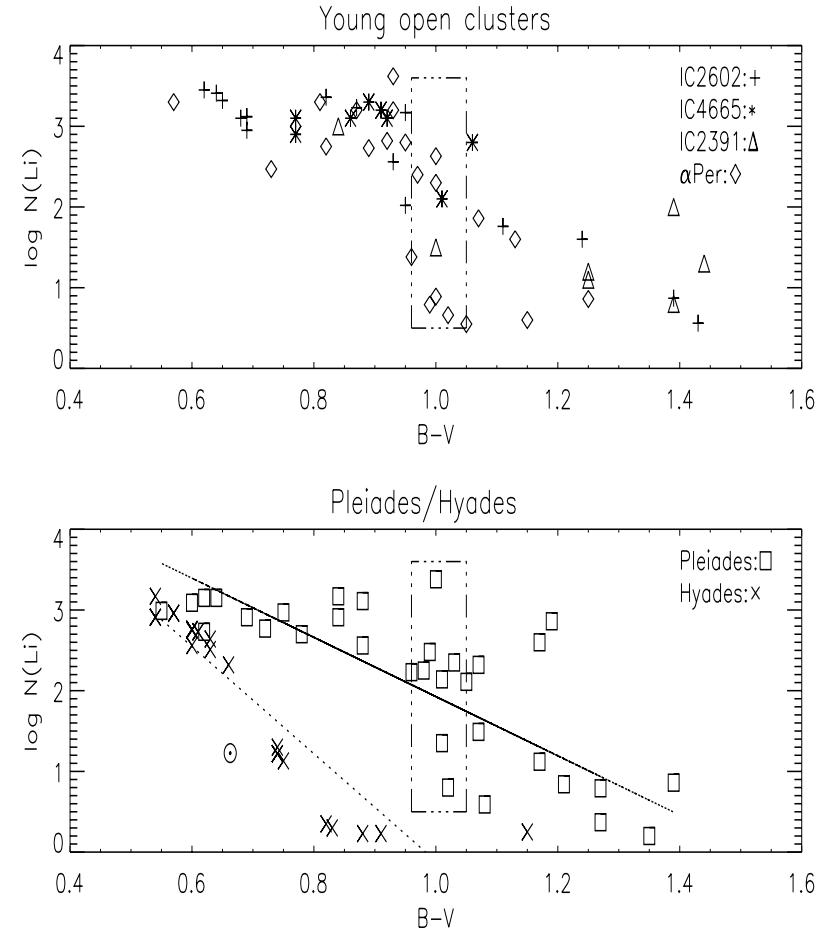

Fig. 3. Li abundance vs. $B-V$. Samples of stars and notation as in Fig. 2. For comparison the Sun $(\odot)$ is included. The boxes mark the abundance spread in $\alpha$ Per and in the Pleiades at $B-V \sim 1$. Lines indicate least-square fits.
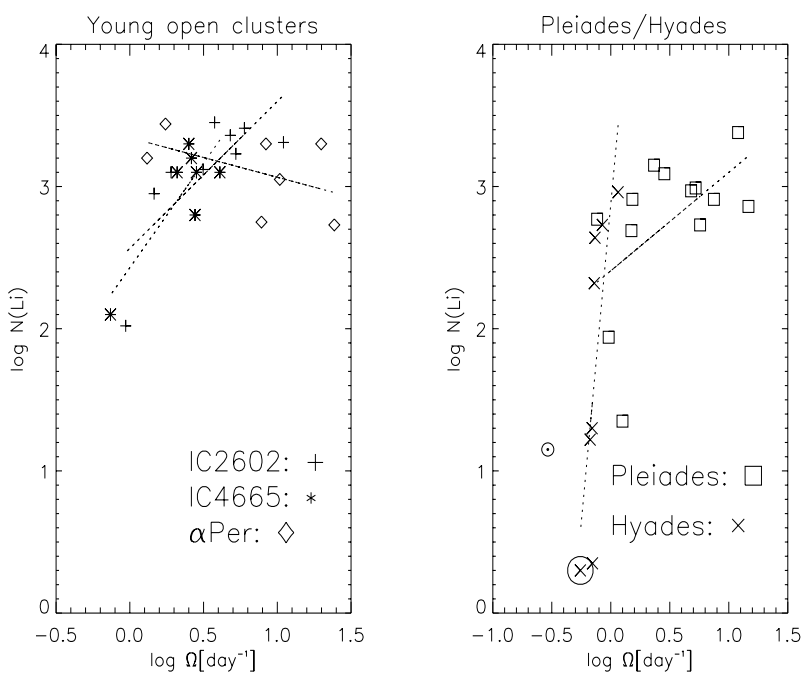

Fig. 4. Li abundances of solar-type stars in open clusters as a function of rotation frequency $\Omega\left(\mathrm{d}^{-1}\right)$. Left: "young" clusters (30-50 Myr). Right: "old" clusters (100-600 Myr). The Sun is indicated by $\odot$, circle means upper limit only.

extinction. Column 1 gives the $V$ magnitude, Col. 2 the $B-V$ color, Col. 3 the rotation period, Col. 4 the Li abundance, and Col. $5 v \sin i$. Spectral types and names are given, too. For detailed references we refer to the ProsserStauffer archive (Prosser \& Stauffer 1998).

In Fig. $4 \mathrm{Li}$ abundance is plotted vs. rotation frequency $\Omega=2 \pi / P_{\text {rot }}$. We cannot state a relation for stars in young open clusters (age $<100 \mathrm{Myr}$ ). The formal linear 

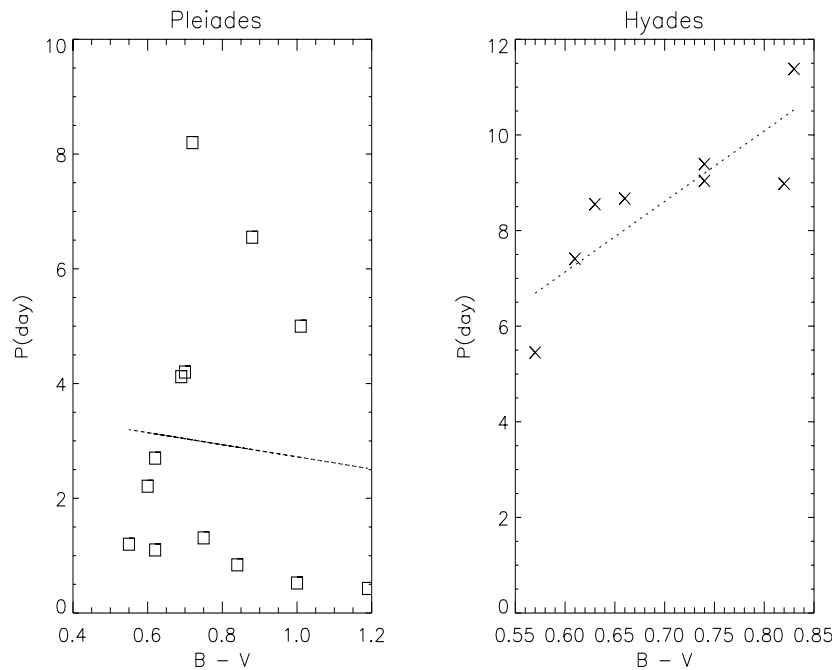

Fig. 5. Rotation period $P_{\text {rot }}$ vs. $B-V$.

least-square fits - shown for IC 2602 and IC 4665 - depend in both cases essentially on one single point. But for stars in "old" clusters (Pleiades, Hyades) the relation looks like existing: the faster the rotation the less the $\mathrm{Li}$ depletion. The lines here have also been derived by least-square linear fits.

Over the selected color range, one expects a $P_{\text {rot }}{ }^{-}$ $(B-V)$ relation as an expression of the well-known $P_{\text {rot }}-$ mass relation. Because of a strong $\mathrm{Li}$ abundance trend with stellar mass, such a correlation could easily simulate a Li-rotation correlation. In fact, Fig. 5 reveals in the Hyades a strong correlation between rotation and color, i.e. between rotation and mass. We conclude, at least in case of the Hyades the $N(\mathrm{Li})-P_{\text {rot }}$ relation is simply an expression of the $N(\mathrm{Li})$-mass relation. In case of the Pleiades no obvious relation exists between rotation and color. Age differences between the Pleiades and Hyades stars may be responsible for the characteristic $N(\mathrm{Li})-P_{\text {rot }}$ differences (Pinsonneault 1997). Nevertheless, one should keep in mind, that a rotational effect on $\mathrm{Li}$ depletion is expected for these "older" Hyades stars, too.

In the case of the Pleiades we have made a twodimensional regression analysis. Provided $\log N(\mathrm{Li})$ depends in a linear manner on $\log \Omega$ as well as on $B-V$, one finds a dependence on the rotation rate which is significant at an one per cent level of error (subject to the condition that the residuals obey a normal distribution with variance not depending on rotation rate and $B-V$ ). The dependence on $B-V$ is significant at 5 per cent only.

What we state is merely a statistical dependence. The assigned "fit" does not necessarily reflect the true physical relation, expected to be far more complex.

\section{Rossby or not Rossby}

One of the important parameters for rotating stars is the inverse Rossby number $\mathrm{Ro}^{-1}$ (the ratio of convective turnover time to the rotation period), which determines
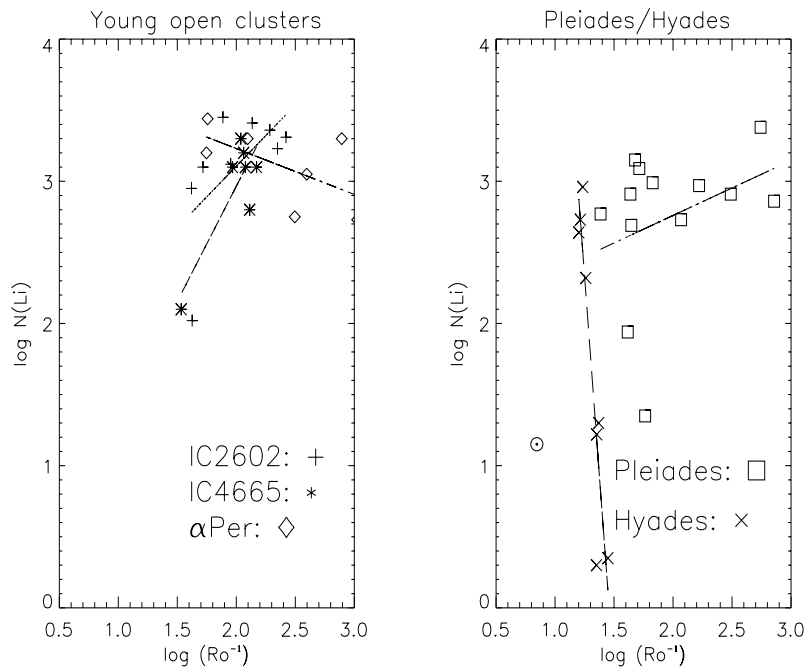

Fig. 6. Solar-type stars in open clusters with Li abundance vs. inverse Rossby number $\mathrm{Ro}^{-1}$. Left: "young" clusters (30-50 Myr). Right: "old" cluster (100-600 Myr). The Sun is indicated by $\odot$.

the time-scale for the Coriolis forces acting on the convectively streaming matter. It is well known that stellar activity as indicated by chromospheric $\mathrm{H}$ and $\mathrm{K}$ emission is closer related with the Rossby number than with the rotation rate itself. Therefore, we examine here whether use of the (inverse) Rossby number instead of the rotation rate improves on the Li-rotation relation or not.

The observed rotation rate is that of the surface. Its dependence on the depth is, of course, unknown. The turnover time $\tau_{\text {corr }}$ (in days) is computed using an empirical relation derived by Noyes et al. (1984),

$\log \tau_{\text {corr }}= \begin{cases}1.362-0.166 x+0.025 x^{2}-5.323 x^{3}, & x>0 \\ 1.362-0.14 x, & x<0\end{cases}$

where $x=1-(B-V)_{0}$.

Using the inverse Rossby number instead of the rotation period does not improve on the Li-rotation relation (Fig. 6), because within the spectral range considered the characteristic turnover time changes by a small factor only. Besides that, this result strengthens our view that Li depletion in solar-type stars is by no means connected with a property of the convection zone but rather with a kind of mixing below that zone, in the tachocline. In fact, both, the Sun's turnover time of about 12 days (Noyes et al. 1984) as well as the convective diffusion time of 100 yr (Rüdiger \& Pipin 2001) are too short to explain the amount of Li depletion found in the Sun.

\section{Spread}

In Fig. 3 the well known spread in Li surface abundance (at $B-V \sim 1$ ) is marked for $\alpha$ Per and the Pleiades. The spread is of the order of $\sim 1.5$ dex. For explanations of this spread see King et al. (2000). The plot of Li abundance vs. rotation period in Fig. 7 hints at a dependence 

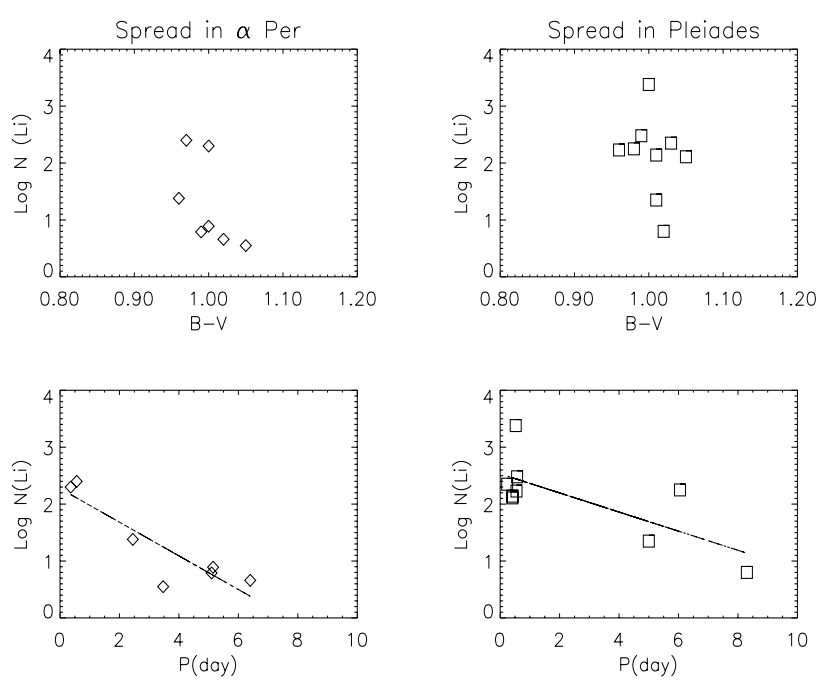

Fig. 7. Li abundances in the $\alpha$ Per Cluster and in the Pleiades for $0.95 \leq B-V \leq 1.05$ (top), and how the Li abundances correlate with the rotation period (bottom).

on rotation: the shorter the rotation period the higher the Li abundance, i.e. the less the Li depletion. Despite the fact that the number of observations is small, the indicated trend seems to be significant. In the case of the nine Pleiades stars with $0.95 \leq B-V \leq 1.05$ the null hypothesis (i.e. no dependence on rotation period) is rejected at a 5 per cent level of error.

We therefore conclude that rotation may play a role for inducing the $\mathrm{Li}$ abundance spread but, obviously, it cannot be the only parameter which is responsible for it.

\section{Discussion}

The Li problem in solar-type stars has observationally two facets: long-term Li depletion in the life of stars as derived from comparing open clusters of different ages, and a real spread in Li abundance for cool main-sequence stars, not accounted for by standard stellar models.

We have limited our discussion of a possible Li-rotation relation to the spectral range $\mathrm{G} 0 \ldots \mathrm{K} 0$ in order to compare similar stellar structures. It is the small number of stars for which Li abundances as well as rotation periods are available that prevents us from making more definitive statements. However, two conclusions seem to be justified by even the present data.

(i) At least for solar-type stars $(\mathrm{G} 0 \ldots \mathrm{K} 0)$ in the Pleiades there is a correlation between $\mathrm{Li}$ abundance and rotation besides the large-scale trend with color. Using rotation periods instead of projected rotational velocities $v \sin i$ confirms: the faster a star rotates the higher in the mean its $\mathrm{Li}$ abundance. We state the absence of such a relation for the young clusters IC 2602, IC 2391, and $\alpha$ Per;

(ii) Despite of the small-number statistics even at fixed color such a correlation between Li abundance and rotation period is indicated in $\alpha$ Per and in the Pleiades.
The Li abundance in a star is convectionally and rotationally affected. Fast rotation seems to suppress Li depletion.

Fast rotation implies magnetic field generation. Stuik et al. (1997) have computed NLTE-models and do not exclude that magnetic activity influences Li-line formation in Pleiades stars. In fact, assumptions about the activitydependent photospheric coverage of spots and plages may affect $\mathrm{Li}$ abundance estimates (Carlsson et al. 1994; King et al. 2000). As a consequence the estimated Li depletion would be fictitious.

Usually one seeks the reason for surface Li depletion in a special transport process in the stellar interior. The location of the Li burning zone depends strongly on mass and age (Fig. 1). That lithium is still present in the Sun after 4.6 Gyr indicates that any non-convective mixing must be slow. Most time scales are much shorter, e.g. convective diffusion time scale $\sim 100 \mathrm{yr}$, rotation period $\sim 26$ days.

Rüdiger \& Pipin (2001) have constructed models of rotation-induced turbulence in the tachocline. Because of the unresolved structure of the tachocline they used parametrized models. According to these models the diffusion of chemicals remains small for rapid rotators due to rotational quenching of horizontal turbulence (rms velocity of $\sim 1 \mathrm{~cm} \mathrm{~s}^{-1}$ ).

The Rossby number is a characteristic feature of the convection zone alone and not a property of the tachocline. Indeed, as Fig. 6 confirms use of the Rossby number instead of the rotation period does not lead to a tighter Li-rotation correlation.

In the case of very young clusters we have not found a corresponding relation between $\mathrm{Li}$ abundance and rotation. The same was stated by Duncan \& Rebull (1996) for the Orion nebula region $(\sim 10 \mathrm{Myr})$. Obviously, the stars have not completed their pre-main sequence evolution (D'Antona \& Mazzitelli 1994).

An enlarged sample of photometrically derived rotation periods of cluster stars with known $\mathrm{Li}$ abundances will clarify the significance of the indicated dependence of Li depletion for late-type main-sequence stars on the rotation period.

Acknowledgements. The authors would like to thank the referee, J. King, for valuable suggestions.

\section{References}

Ahrens, B., Stix, M., \& Thorn, M. 1992, A\&A, 264, 673

Allain, S., Bouvier, J., Prosser, C., et al. 1996, A\&A, 305, 498

Allen, C. W. 1991, Astrophys. Quant. (Athlone Press, London)

Balachandran, S., Lambert, D. L., \& Stauffer, J. R. 1988, ApJ, 333, 267

Balachandran, S., Lambert, D. L., \& Stauffer, J. R. 1996, ApJ, 470, 1243

Barnes, J. R., Cameron, A. C., Unruh, Y. C., et al. 1998, MNRAS, 299, 904

Blöcker, T., Holweger, H., Freytag, B., et al. 1999, Space Sci. Rev., 85, 105

Boubnov, B. M., \& Golitsin, G. S. 1995, Convection in Rotating Fluids (Kluwer Academic Publishers, Dordrecht, Boston, London) 
Carlsson, M., Rutten, R. J., Bruls, J. H. M. J., et al. 1994, A\&A, 288, 860

D'Antona, F., \& Mazzitelli, I. 1994, ApJS, 90, 467

Deliyannis, C. P., King, J. R., Boesgaard, A. M., et al. 1994, ApJ, 434, L71

Duncan, D. K., \& Rebull, L. M. 1996, PASP, 108, 738

García López, R. J., Rebolo, R., \& Martín, E. L. 1994, A\&A, 282,518

Jones, B. F., Fischer, D., \& Shetrone, M. 1997, AJ, 114, 352

King, J. R., Krishnamurthi, A., \& Pinsonneault, M. H. 2000, AJ, 119, 859

Krishnamurthi, A., Pinsonneault, M. H., Barnes, S., et al. 1997, ApJ, 480, 303

Krishnamurthi, A., Terndrup, D. M., Pinsonneault, M. H., et al. 1998, ApJ, 493, 914

Livshits, M. A. 1997, Solar Phys., 173, 377

Noyes, R. W., Hartmann, L. W., Baliunas, S. L., et al. 1984, ApJ, 279, 763

Pinsonneault, M. 1997, ARA\&A, 35, 557

Prosser, C. F. 1992, AJ, 103, 488

Prosser, C. F., \& Stauffer, J. R. 1998, ftp: //cfa0.harvard.edu/pub/stauffer

Prosser, C. F., Schild, R. E., Stauffer, J. R., et al. 1993a, PASP, 105,269
Prosser, C. F., Shetrone, M. D., Marilli, E., et al. 1993b, PASP, 105, 1407

Randich, S., Aharpour, N., Pallavicini, R., et al. 1997, A\&A, 323,86

Randich, S., Martín, E. L., García López, R. J., et al. 1998, A\&A, 333, 591

Rüdiger, G., \& Pipin, V. V. 2001, A\&A, 375, 149

Ryan, S. G., \& Deliyannis, C. P. 1995, ApJ, 453, 819

Schlattl, H., \& Weiss, A. 1999, A\&A, 347, 272

Soderblom, D. R., Jones, B. F., Balachandran, S., et al. 1993, AJ, 106, 1059

Stauffer, J. R., Hartmann, L. W., Prosser, C. F., et al. 1997, ApJ, 479, 776

Stix, M., \& Skaley, D. 1990, A\&A, 232, 234

Stuik, R., Bruls, J. H. M. J., \& Rutten, R. J. 1997, A\&A, 322, 911

Thorburn, J. A., Hobbs, L. M., Deliyannis, C. P., et al. 1993, ApJ, 415, 150

Vincent, A., Michaud, G., \& Meneguzzi, M. 1996, Phys. Fluids, 8,1312

Zahn, J.-P. 1992, A\&A, 265, 115 